\title{
Long Noncoding RNA AF131217.1 Regulated Coronary Slow Flow-Induced Inflammation Affecting Coronary Slow Flow via KLF4
}

Haibing Jiang ${ }^{1 \#}$, MD, Zhengrong Ge "1, MD; Lijing Zhang ${ }^{1}$, MD; Yi Yang ${ }^{1}$, MD; Xueqin Zhai ${ }^{1}$, MD; Zhanxi Chen ${ }^{1}$, MD; Qing Wei', MD

\section{ABSTRACT}

Introduction: This study investigated the correlation between the levels of long noncoding ribonucleic acids (IncRNAs) AF131217.1 and coronary slow flow (CSF).

Methods: A total of 22 patients in the high-sensitivity C-reactive protein (hsCRP) group diagnosed with CSF from January 2018 to December 2018 were enrolled in this study. Coronary flow velocity was determined using the thrombolysis in myocardial infarction frame count (TFC) method. Results: LncRNA AF131217.1 expression in the CSF model was activated. Mean TFC was positively correlated with IncRNA AF131217.1 levels and hsCRP levels.
\end{abstract}

LncRNA AF131217.1 induced inflammation factor levels in the in vitro model. Micro ribonucleic acid (miR)-128-3p is a target spot of IncRNA AF131217.1 on the inflammation in vitro model via Kruppel-like factor (KLF) 4. MiR-128-3p reduced inflammation factor levels (tumor necrosis factor alpha, interleukin [IL]-6, IL-1 $\beta$, and IL-18). Conclusion: Thus, IncRNA AF131217.1 promoted inflammation in the regulated CSF via KLF4 by miR-128-3p.

Keywords: Myocardial Infarctation. Inflammation. Interleukin-18. Interleukin-6. RNA, Long Noncoding. C-Reactive Protein. MicroRNAs. GKLF protein.

\begin{tabular}{|c|c|c|c|}
\hline \multicolumn{4}{|c|}{ Abbreviations, acronyms \& symbols } \\
\hline ACS & $=$ Acute coronary syndrome & LncRNAs & $=$ Long noncoding ribonucleic acids \\
\hline C/EBP $\beta$ & = CCAAT/enhancer binding protein beta & LPS & = Lipopolysaccharide \\
\hline CDNA & $=$ Complementary deoxyribonucleic acid & MiR & $=$ Micro ribonucleic acid \\
\hline CSF & $=$ Coronary slow flow & mRNA & $=$ Messenger ribonucleic acid \\
\hline ELISA & = Enzyme-linked immunosorbent assay & Mut & $=$ Mutant \\
\hline GAPDH & = Glyceraldehyde-3-phosphate dehydrogenase & NF-кB & = Nuclear factor kappa B \\
\hline hsCRP & $=$ High-sensitivity C-reactive protein & RNA & $=$ Ribonucleic acid \\
\hline HUVECS & $=$ Human umbilical vein endothelial cells & TFC & $=$ Thrombolysis in myocardial infarction frame count \\
\hline & $=$ Interleukin & TNF- $a$ & $=$ Tumor necrosis factor alpha \\
\hline KLF & = Kruppel-like factors & WT & $=$ Wild type \\
\hline
\end{tabular}

\section{INTRODUCTION}

The coronary slow flow (CSF) phenomenon is defined as the presence of delayed perfusion of peripheral coronary artery identified by coronary arteriography after excluding coronary artery disease, cardiomyopathy, valvular disease, congenital heart disease, connective tissue disease, etc. Although coronary

'Department of Cardiology, Affiliated Chinese Medicine Hospital of Xinjiang Medical University, Urumqi, Xinjiang, People's Republic of China.

\section{\#Contributed equally.}

This study was carried out at the Affiliated Chinese Medicine Hospital of Xinjiang Medical University, Urumqi, Xinjiang, People's Republic of China. angiography in patients with slow blood flow shows no stenosis of coronary artery, recurrent cardiovascular events still occur, generally clinically manifested by angina, arrhythmia, or acute coronary syndrome (ACS), which may be associated with CSF and usually requires emergency admission ${ }^{[1,2]}$. The exact pathological

Correspondence Address:

Haibing Jiang

(iD) https://orcid.org/0000-0002-9716-1921

Department of Cardiology, Affiliated Chinese Medicine Hospital of Xinjiang Medical University

No. 116 Huanghe Road, Urumqi, Xinjiang, People's Republic of China - Zip Code: 830000 E-mail: haibingjiang@tom.com 
mechanism of CSF is still unclear. At present, there is no standardized therapeutic measures for CSF in clinical practice, with inconsistent reported efficacy of drug treatment ${ }^{[2]}$. Studies have shown that CSF is strongly associated with cardiovascular adverse events, such as arrhythmia, ACS, and sudden cardiac death. In addition, the levels of high-sensitivity C-reactive protein (hsCRP), vascular cell adhesion molecules, and intercellular adhesion molecules are significantly increased in CSF patients than in healthy individuals, which is also positively correlated with thrombolysis in myocardial infarction frame count (TFC), indicating endothelial activation and inflammatory response in CSF patients ${ }^{[2,3]}$.

Kruppel-like factors (KLF) are a type of transcription factors with zinc-finger structure, which is characterized by the three $\mathrm{C} 2 \mathrm{H} 2$ zinc-finger structures at the carboxyl terminal[ ${ }^{[4]}$. KLF family members are widely involved in the regulation of multiple life activities, including cell proliferation, apoptosis, differentiation, and embryonic development ${ }^{[5]}$. The abnormal function of KLF family members is strongly associated with metabolic diseases, cardiovascular diseases, and cancer. KLF4, originally isolated from the gastrointestinal tract, is one of the transcriptional regulators required for the early differentiation of adipocytes ${ }^{[6]}$. It activates the expression of the CCAAT/enhancer binding protein beta (C) $E B P \beta)$ gene by binding itself to the promoter of $C / E B P \beta$, thereby activating the downstream cascade of fat cell differentiation to promote this differentiation ${ }^{[6,7]}$. In recent years, KLF4 has become a research hotspot in relevant fields due to its regulatory roles on chronic inflammatory responses of various cells ${ }^{[4]}$.

Long noncoding ribonucleic acids (IncRNAs) are a type of noncoding RNA with over 200 bp in length ${ }^{[8]}$. To date, hundreds of thousands of eukaryotic IncRNAs have been found, most of which are lowly conservative and involved in the pathogenesis and progression of a series of diseases, including tumors, nervous system disorders, metabolic diseases, reproductive development, and cardiovascular diseases ${ }^{[9]}$. Long intergenic noncoding RNA-p21, a p53-induced IncRNA, is able to inhibit cell proliferation and promote apoptosis during atherosclerosis $s^{[9,10]}$. This study investigated the correlation between the levels of IncRNA AF131217.1 and CSF.

\section{METHODS}

\section{Study Subjects and CSF Diagnosis}

A total of 22 patients in the hsCRP group diagnosed with CSF at the Affiliated Chinese Medicine Hospital of Xinjiang Medical University, from January 2018 to December 2018, were enrolled in this study. All patients had stenosis of lumen diameter < 40\% (no significant vascular lesion). The control group was 24 patients who did not meet the abovementioned criteria. All procedures performed in studies involving human participants were in accordance with the ethical standards of the institutional and/or National Research Committee and with the 1964 Helsinki Declaration and its later amendments or comparable ethical standards. This study was approved by the Ethics Committee of the Affiliated Chinese Medicine Hospital of Xinjiang Medical University. Written informed consent was obtained from all individual participants included in the study. TFC was used to quantitatively measure coronary blood flow using a cineangiography. CSF was diagnosed if TFC $>27$ in at least one coronary artery. Three cardiologists who were blinded to the clinical findings independently assessed the TFC.

\section{LncRNA and micro RNA Analysis}

Total RNA was purified with the RNeasy Kit (Qiagen). Complementary deoxyribonucleic acid (cDNA) and complementary RNA were generated and hybridized to HumanHT-12 v4 BeadChips (Illumina). Cubic spline-normalized (without background normalization) data were analyzed by the NIA Array Analysis tool (http://lgsun.grc.nia.nih.gov/ANOVA).

\section{Luciferase Reporter Assay}

Micro RNA (miR)-128-3p was inserted into the luciferase gene psiCHECK2 vector, named as KLF4-WT (the wild type) or KLF4Mut (mutant) 3'-UTR. Next, miR-128-3p was inserted into the luciferase gene psiCHECK2 vector, named as IncRNA AF131217.1WT or IncRNA AF131217.1-Mut 3'-UTR. After transduction, at 48 hours, cell was transduced using Lipofectamine ${ }^{\circledR} 2000$ Reagent (Thermo Fisher Scientific, Inc.). The luciferase assay was performed using Luciferase Reporter Gene Detection Kit (SigmaAldrich Co., St. Louis, Missouri, United States of America).

\section{Real-Time Reverse Transcription Polymerase Chain Reaction}

Total RNA was isolated with the RNeasy Micro Kit (Qiagen) and cDNA was synthesized with the Maxima First Strand cDNA Synthesis Kit. Quantitative real-time polymerase chain reaction was performed using SYBR Green Master Mix. Relative fold changes were calculated using the $2^{-\Delta \Delta \mathrm{Ct}}$ method.

\section{Cell Culture and Treatment}

Human umbilical vein endothelial cells (HUVECS) were cultured in Roswell Park Memorial Institute 1640 medium (Thermo Fisher Scientific, Inc.) supplemented with 10\% fetal bovine serum (Thermo Fisher Scientific, Inc.) at $37^{\circ} \mathrm{C}$ with $5 \%$ $\mathrm{CO}_{2}$. HUVECS $\left(1 \times 10^{5} /\right.$ well) were transiently transfected with 50 nM of IncRNA AF131217.1, silencing IncRNA AF131217.1, miR128-3p, and negative control using Lipofectamine ${ }^{\circledast} 2000$ reagent (Invitrogen; Thermo Fisher Scientific, Inc.). After 48 hours of transfection, HUVECs were treated with $100 \mu \mathrm{g} / \mathrm{ml}$ oxidized lowdensity lipoprotein for 24 hours.

\section{Western Blots}

Cells were lysed with radioimmunoprecipitation buffer, and protease and phosphatase inhibitors (Sigma-Aldrich) were added. Protein concentration was measured using a bicinchoninic acid protein assay kit (Beyotime, Jiangsu, China). Equal amounts of proteins were separated by 10\% sodium dodecyl sulphate-polyacrylamide gel electrophoresis and transferred to polyvinylidene fluoride membranes. After blocking with no-fat skim milk, the membranes were incubated with primary antibodies (KLF4, RhoF, nuclear factor kappa B [NF-KB], and glyceraldehyde-3-phosphate dehydrogenase) diluted in Tris Buffered Saline with Tween 20 overnight at $4^{\circ} \mathrm{C}$ and then with 
the horseradish peroxidase-conjugated secondary antibody for two hours at room temperature. Protein bland was detected by electrochemiluminescence chromogenic substrate kits and quantified by Image Lab 3.0 (Bio-Rad Laboratories, Inc.).

\section{Enzyme-linked Immunosorbent Assay (ELISA) Kit Analysis}

Serum samples were collected at $1000 \mathrm{~g}$ for $10 \mathrm{~min}$ and used to measure tumor necrosis factor alpha (TNF-a), interleukin (IL)-6, $\mathrm{IL}-1 \beta$, and IL-18 levels. Cell samples were also collected at 1000 $\mathrm{g}$ for $10 \mathrm{~min}$ and used to measure TNF- $\alpha, I L-6, I L-1 \beta$, and IL-18 levels. TNF-a, IL-6, IL-1 $\beta$, and IL-18 ELISA kits were purchased from Nanjing Jiancheng Biological Engineering Research Institute Co. LTD (Nanjing, China).

\section{Statistical Analysis}

All data are expressed as the mean \pm standard error of mean. $P<0.05$ was considered statistically significant. Statistical significance between groups of data was calculated by Student's t-test or one-way analysis of variance and Tukey's post-test.

\section{RESULTS}

\section{LncRNA AF131217.1 Expression in the CSF Model}

Initially, we sought to validate IncRNA AF131217.1 expression in the CSF model. We found using gene chip that six genes were downregulated and 11 genes were upregulated in the CSF model (Figures 1A and 1B). LncRNA AF131217.1 expression in the CSF model was activated, compared with the control group (Figure 1C). Mean TFC was positively correlated with IncRNA AF131217.1 levels (Figure 1D) and hsCRP levels (Figure 1E).

\section{LncRNA AF131217.1 Regulated Inflammation in the In Vitro Model}

Moreover, in the lipopolysaccharide (LPS)-induced in vitro model, IncRNA AF131217.1 plasmid increased IncRNA AF131217.1 expression and induced inflammation factor levels (TNFa, IL-6, IL-1 $\beta$, and IL-18) (Figures 2A to 2E). Then, in LPSinduced in vitro model, silencing IncRNA AF131217.1 decreased IncRNA AF131217.1 expression and reduced inflammation factor levels (TNFa, IL-6, IL-1 $\beta$, and IL-18) (Figure 2F to 2J). Thus, IncRNA
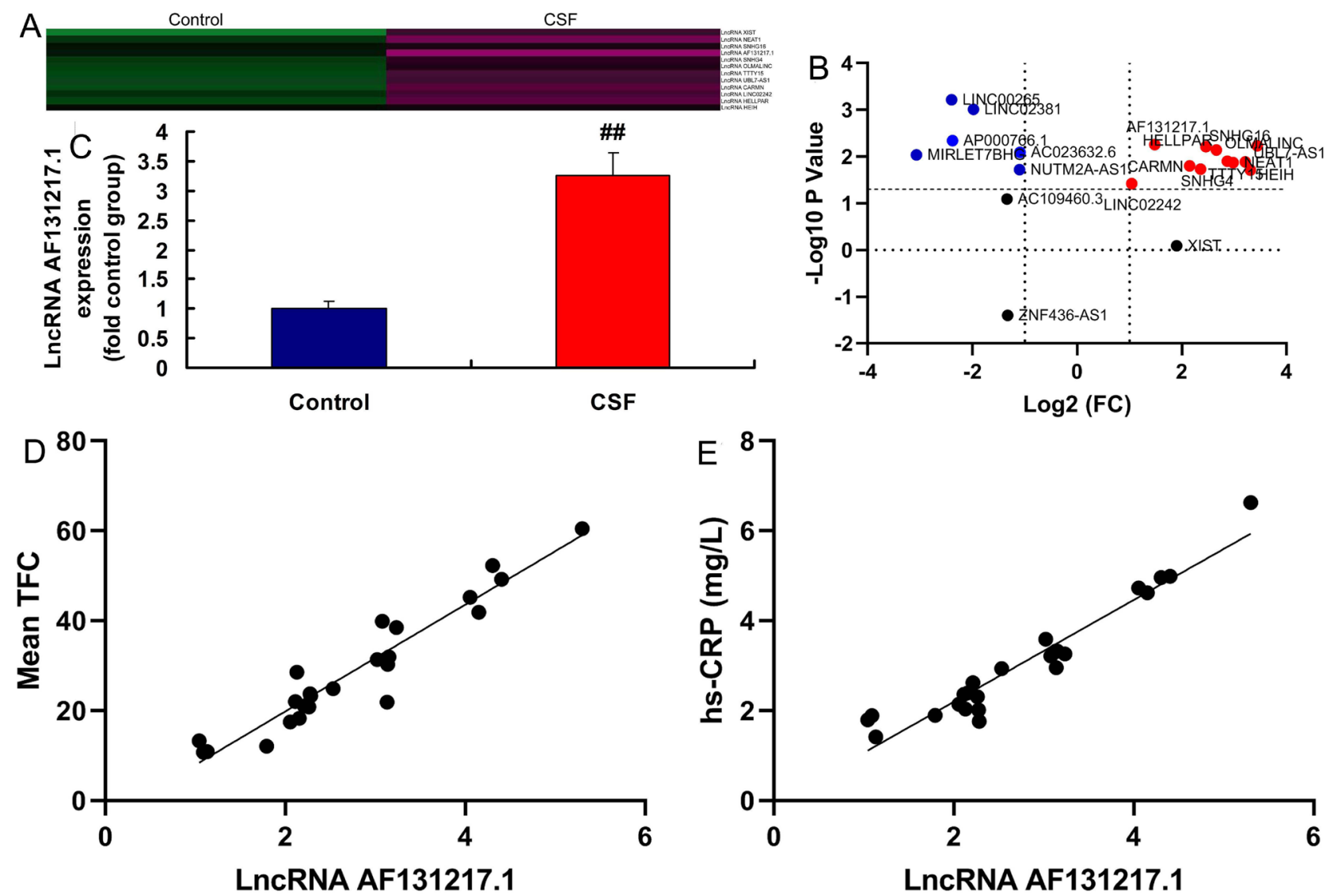

Fig. 1 - Long noncoding ribonucleic acid (InCRNA) AF131217.1 expression in the coronary slow flow (CSF) model. Heat map and volcano figure of gene chip (A and B), quantitative real-time polymerase chain reaction of IncRNA AF131217.1 expression (C) in the CSF model. The mean thrombolysis in myocardial infarction frame count (TFC) was positively correlated with InCRNA AF131217.1 levels (D) and high-sensitivity C-reactive protein (hsCRP) levels (E). Control, normal volunteer group; $h s C R P$, hsCRP patients group. ${ }^{\#} P<0.01$ compared with normal volunteer group. 

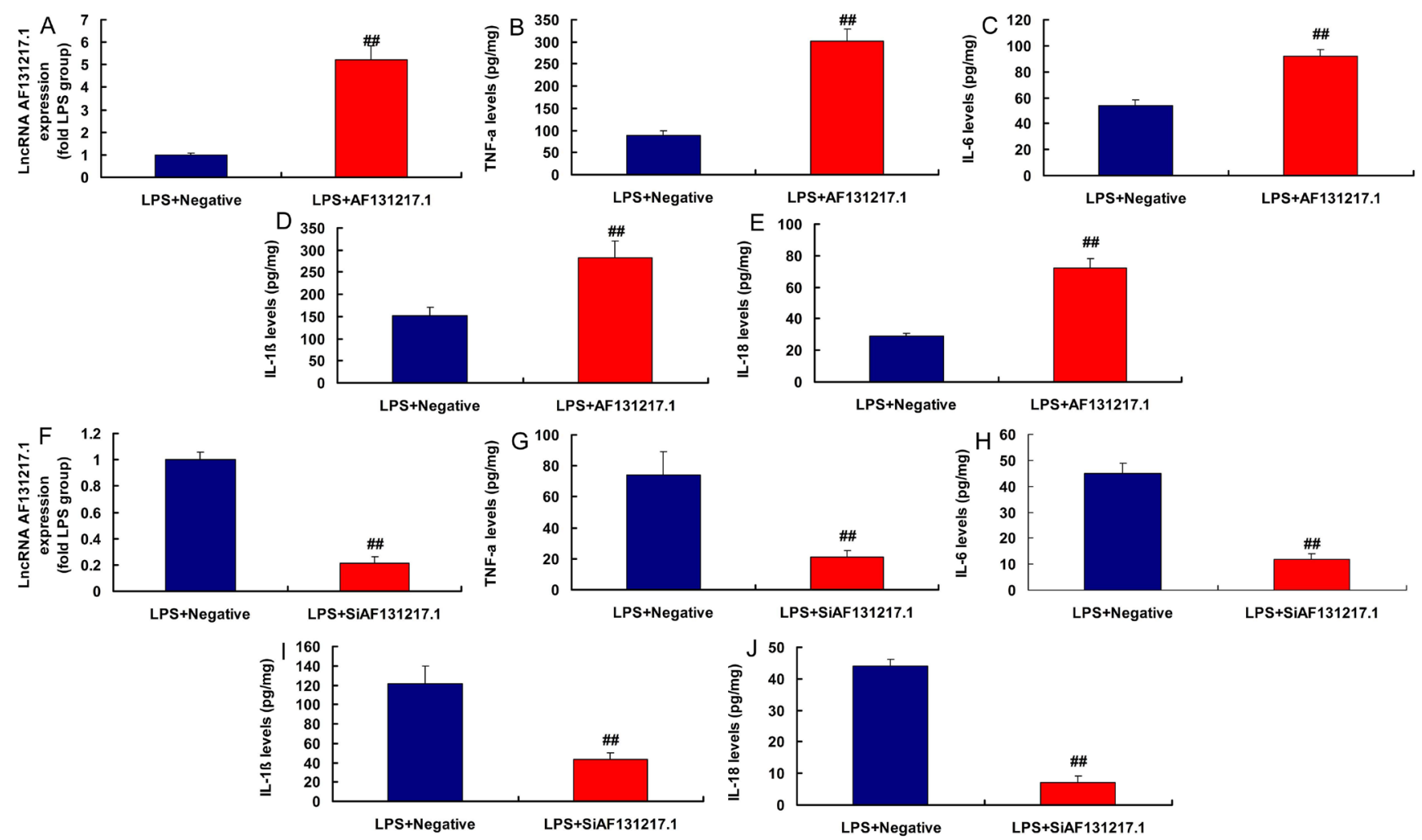

Fig. 2 - Long noncoding ribonucleic acid (InCRNA) AF131217.1 regulated inflammation in the in vitro model. LncRNA AF131217 expression (A), tumor necrosis factor alpha (TNF-a), interleukin (IL)-6, IL-1 $\beta$, and IL-18 (B, C, D, and E) in overexpression of IncRNA AF131217 group; IncRNA AF131217 expression (F), TNF- $\alpha, I L-6, I L-1 \beta$, and IL-18 (G, H, I, and J) in downregulation of IncRNA AF131217 group. LPS+Negative, LPSinduced in vitro model by negative mimics group; LPS+AF131217.1, LPS-induced in vitro model by AF131217.1 group. ${ }^{* \# P} P 0.01$ compared with LPS+Negative group. LPS=lipopolysaccharide.

AF131217.1 expression in the CSF model was upregulated and started an inflammatory reaction.

\section{MiR-128-3p is a Target Spot of IncRNA AF131217.1 on the Inflammation In Vitro Model}

In our search to define the possible target spot of IncRNA AF131217.1 on inflammation of CSF, miR-128-3p expression had a negative correlation with InCRNA AF131217.1 levels in the CSF model (Figure 3A). The relative luciferase activity in AF131217.1-WT and miR-128-3p groups was lower than that of AF131217.1-Mut and miR-NC cotransfection groups (Figures 3B and 3C). Nonetheless, overexpression of IncRNA AF131217.1 suppressed miR-128-3p expression and downregulation of IncRNA AF131217.1 induced miR-128-3p expression in LPS-induced in vitro model (Figures 3D and 3E).

\section{MiR-128-3p and KLF4 Expressions in the CSF Model}

In the rat model of CSF, miR-128-3p expression was downregulated and KLF4 expression was upregulated, compared with the control group (Figures $4 \mathrm{~A}$ and $4 \mathrm{~B}$ ). MiR- 128-3p expression had a negative correlation with KLF4 levels in the rat model (Figure 4C). Moreover, in LPS-induced in vitro model, miR-128-3p plasmid increased miR-128-3p expression and reduced inflammation factor levels (TNFa, IL-6, IL-1 $\beta$, and IL-18) (Figures 5A to 5E). Then, in LPS-induced in vitro model, simiR-128-3p AF131217.1 decreased miR-128-3p expression and promoted inflammation factor levels (TNFa, IL-6, IL-1 $\beta$, and IL-18) (Figures $5 \mathrm{~F}$ to $5 \mathrm{~J}$ ). Thus, miR-128-3p expression in the CSF model was downregulated and revealed anti-inflammatory effect.

\section{KLF4 is a Target Spot of miR-128-3p on the Inflammation In Vitro Model}

The study defined the possible target spot of miR-128-3p on inflammation of CSF, the relative luciferase activity in KLF4-WT and miR-128-3p groups was lower than that of KLF4-Mut and miR-NC cotransfection groups (Figures 6A and 6B). Nonetheless, overexpression of miR-128-3p suppressed KLF4, RhoF, and NF$-\mathrm{KB}$ protein expressions (Figures $6 \mathrm{C}$ to $6 \mathrm{E}, 6 \mathrm{6I}$ ). Downregulation of miR-128-3p induced KLF4, RhoF, and NF-KB protein expressions in LPS-induced in vitro model (Figures $6 \mathrm{~F}$ to $6 \mathrm{H}, 6 \mathrm{~J}$ ). 


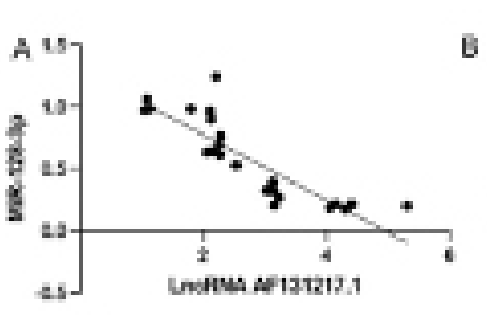

$\mathbf{3}$

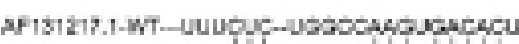

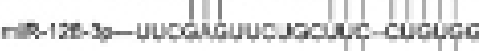

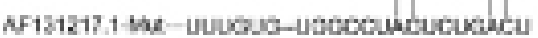
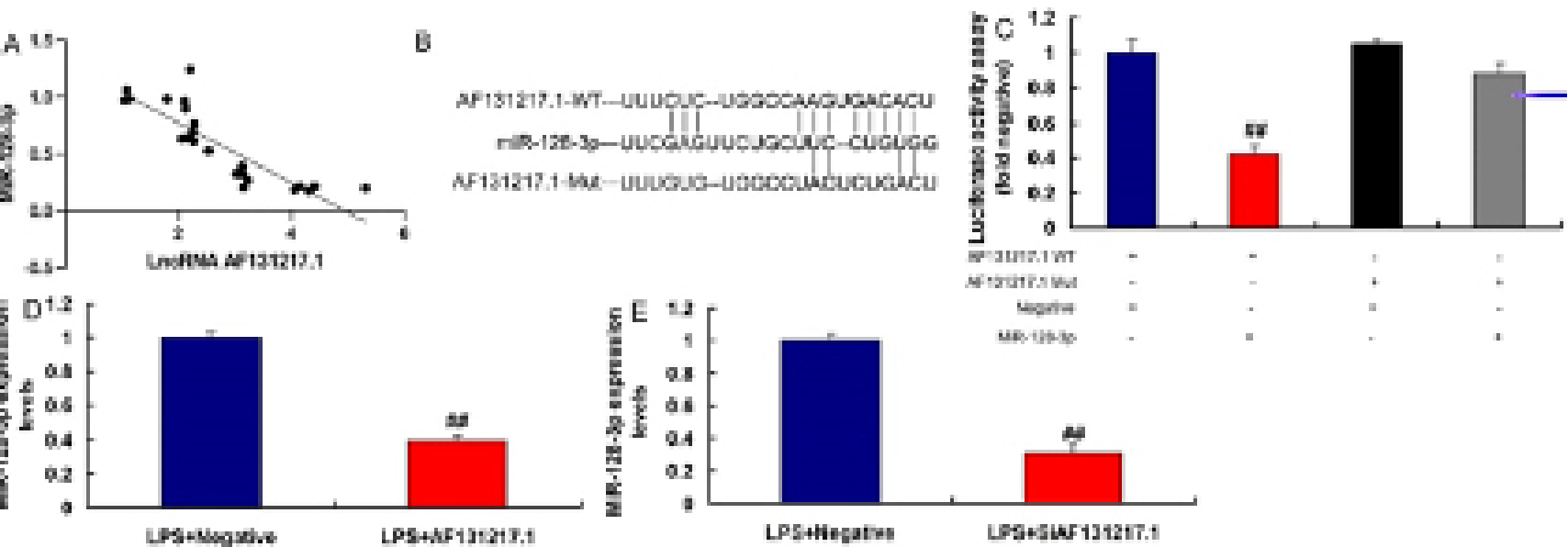

Fig. 3 - Micro ribonucleic acid (miR)-128-3p is a target spot of long noncoding ribonucleic acid (IncRNA) AF131217.1 on the inflammation in vitro model. MiR-128-3p expression had a negative correlation with IncRNA AF131217.1 levels in the coronary slow flow model (A). The luciferase reporter plasmid containing wild type (WT) or mutant (Mut) AF131217.1 was cotransfected with miR-128-3p (B and C), overexpression of IncRNA AF131217.1 suppressed miR-128-3p expression (D), downregulation of InCRNA AF131217.1 induced miR-128-3p expression in lipopolysaccharide (LPS)-induced in vitro model (E). LPS+Negative, LPS-induced in vitro model by negative mimics group; LPS+AF131217, LPSinduced in vitro model by AF131217.1 group. ${ }^{\# P}<0.01$ compared with LPS+Negative group.
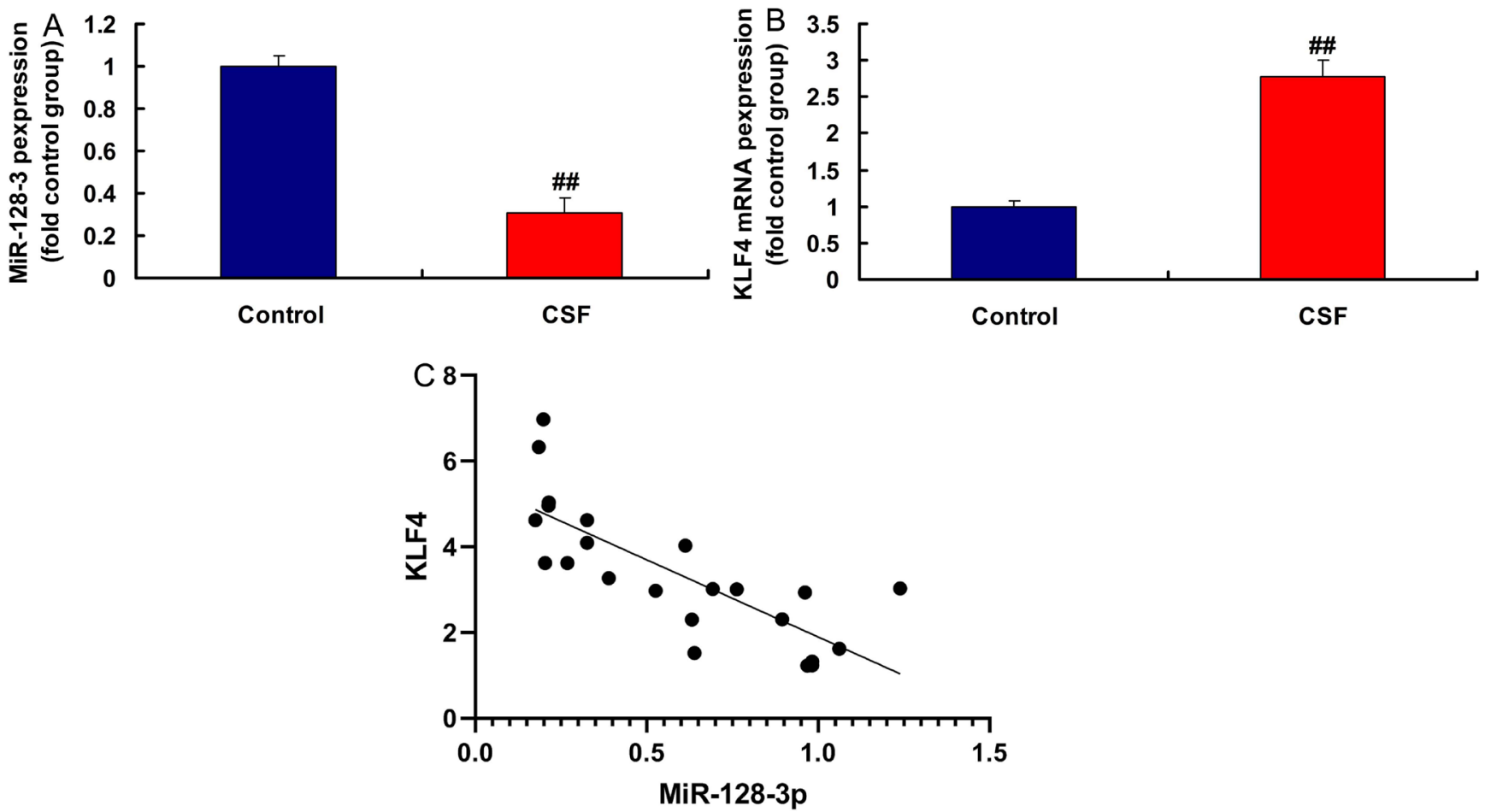

Fig. 4 - Micro ribonucleic acid (MiR)-128-3p and Kruppel-like factor (KLF) 4 expressions in the coronary slow flow (CSF) model (A and B), miR128-3p expression had a negative correlation with KLF4 levels in the rat model (C). Control, normal volunteer group; CSF, CSF patients group. ${ }^{\# \# P}<0.01$ compared with normal volunteer group. $m R N A=$ messenger ribonucleic acid 


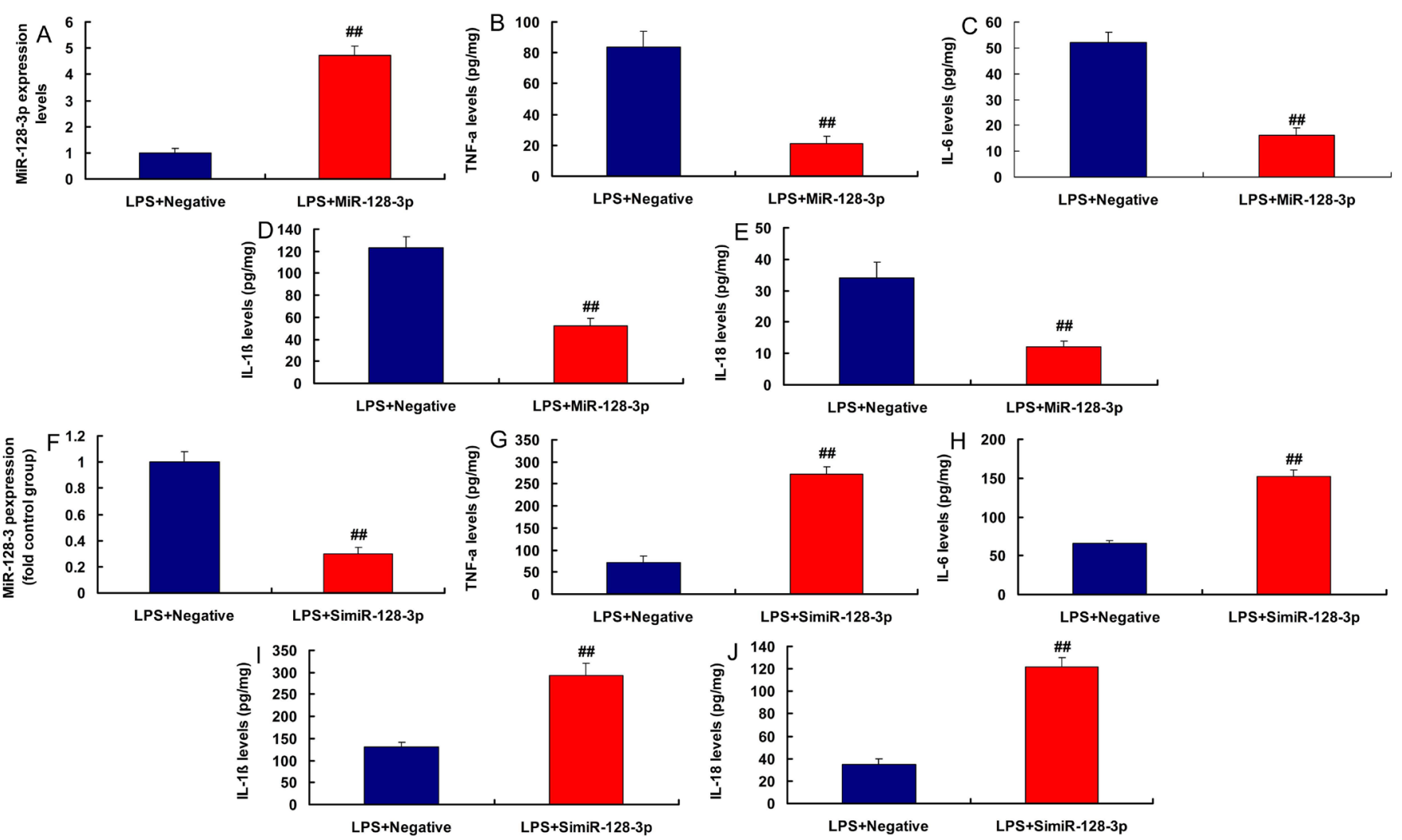

Fig. 5 - Micro ribonucleic acid (MiR)-128-3p regulated inflammation in the in vitro model. MiR-128-3p expression (A), tumor necrosis factor alpha (TNF-a), interleukin (IL)-6, IL-1 $\beta$, and IL-18 (B, C, D, and E) in overexpression of miR-128-3p group; miR-128-3p expression (F), TNF-a, IL-6, $I L-1 \beta$, and IL-18 (G, H, I, and J) in downregulation of miR-128-3p group. LPS+Negative, LPS-induced in vitro model by negative mimics group;

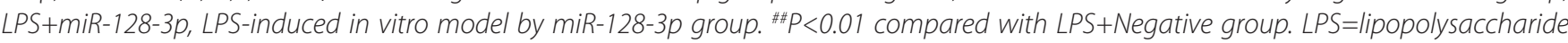

\section{LncRNA AF131217.1 Regulated Inflammation in the In Vitro Model via KLF4 by miR-128-3p}

In an attempt to delineate whether miR-128-3p was required for IncRNA AF131217.1 induction of KLF4 signaling in CSF, we found that miR-128-3p overexpression induced miR-128-3p expression and reduced inflammation factor levels (TNFa, IL-6, IL$1 \beta$, and IL-18) in the in vitro model of IncRNA AF131217.1 (Figure 7A-7E). Meanwhile, miR-128-3p overexpression suppressed KLF4, RhoF, and NF-KB protein expressions in the in vitro model of IncRNA AF131217.1 (Figure 7F-7I).

\section{DISCUSSION}

CSF is defined as the presence of delayed perfusion of peripheral coronary artery but without obvious lesions in the coronary arteries diagnosed by coronary arteriography after excluding coronary spasm, coronary artery dilatation, coronary angioplasty, cardiomyopathy, heart valvular disease, autoimmune diseases, tumors, and other important organs or systemic diseases. More and more attention are being paid to the research of CSF, however, the etiology and pathogenesis of CSF remain unclear ${ }^{[11-13]}$. We showed that IncRNA AF131217.1 expression in the CSF model was activated. Mean TFC was positively correlated with IncRNA AF131217.1 levels and hsCRP levels. These characteristics suggest that IncRNA AF131217.1 participated in the pathophysiological process of CSF.

Lu et al. ${ }^{[14]}$ showed that shear-sensitive IncRNA AF131217.1 reduced inflammation in HUVECs via regulation of KLF4.

Cardiovascular disease is a common disease that threatens the health of human beings, especially the elderly ${ }^{[15]}$. The annual number of people dying from cardiovascular disease ranks the first globally ${ }^{[15]}$. LncRNAs are a type of noncoding RNAs with over $200 \mathrm{nt}$ in length, which are also the most abundantly expressed $\mathrm{RNA}^{[16,17]}$. Accumulative studies have revealed that IncRNAs play an important regulatory role in the pathogenesis and development of cardiovascular diseases ${ }^{[16]}$. Interestingly, IncRNA AF131217.1 induced inflammation factor levels and played an important role in the inflammation of CSF.

Wang et al. ${ }^{[18]}$ showed that miR-128-3p accelerates cardiovascular calcification in type 2 diabetes mellitus rats. Studies have shown that CSF is closely associated with cardiovascular adverse events, including arrhythmia, ACS, and sudden cardiac death ${ }^{[1,20]}$. When coronary angiography is performed on patients with suspected cardiovascular disease, the detection rate of CSF is approximately $1 \%^{[19]}$. Recent studies have demonstrated that inflammatory response plays an important role in the pathogenesis 
A

KLF-WT---AGACAGUCUGUUAUGCACUGUGG miR-128-3p---UUUCUCUGGCCAAGUGACACU KLF-Mut---AGACAGUCUGUUAUGGUCGGUGG
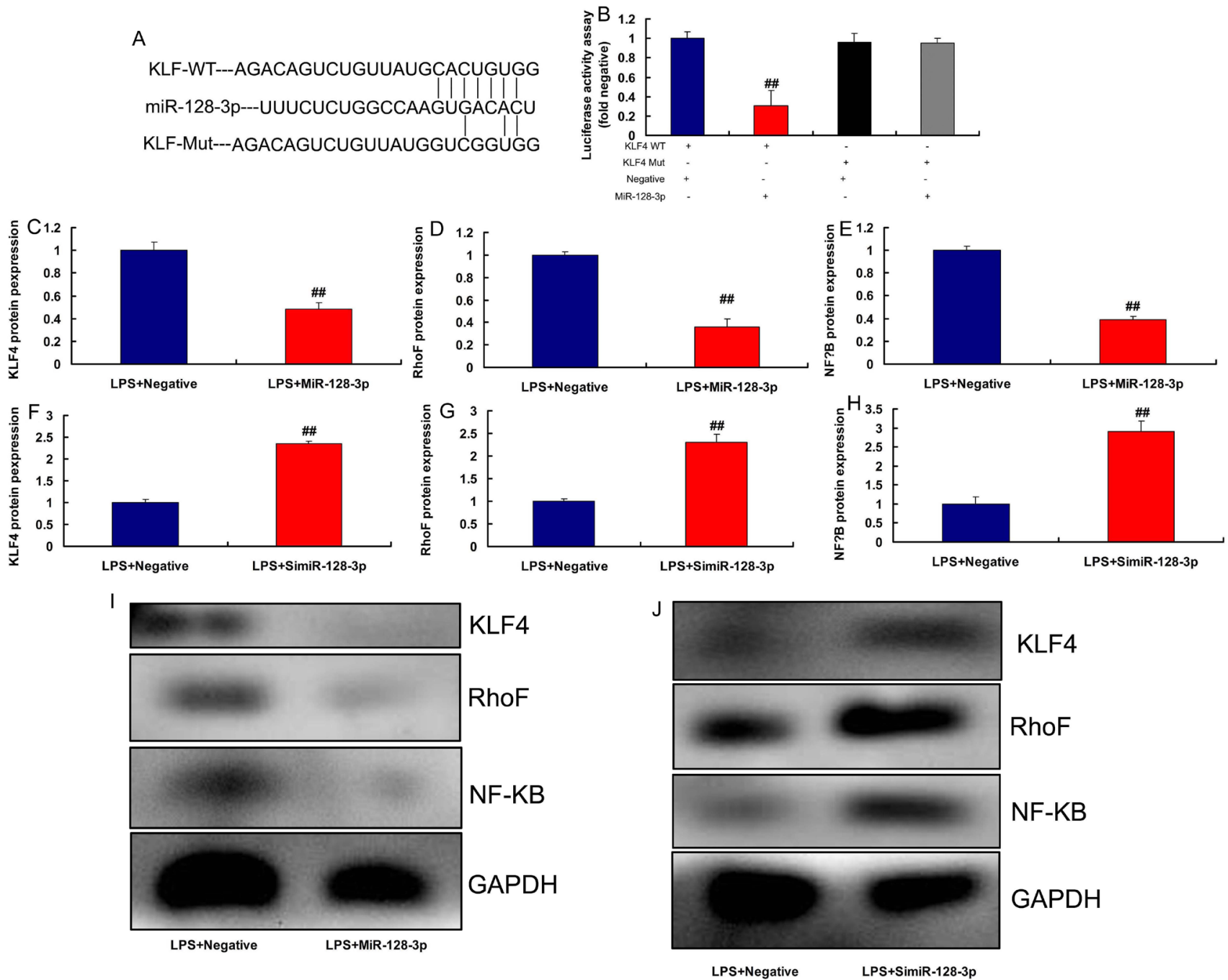

Fig. 6 - Kruppel-like factor (KLF) 4 is a target spot of micro ribonucleic acid (MiR)-128-3p on the inflammation in vitro model. The luciferase reporter plasmid containing wild type (WT) or mutant (Mut) KLF4 was cotransfected with miR-128-3p (A and B), KLF4, RhoF, and nuclear factor kappa B (NF-KB) protein expressions (C, D, E, and I) by overexpression of miR-128-3p; KLF4, RhoF, and NF-KB protein expressions (F, G, H, and J) by downregulation of miR-128-3p. LPS+Negative, LPS-induced in vitro model by negative mimics group; LPS+miR-128-3p, LPS-induced in vitro model by miR-128-3p group; LPS+simiR-128-3p, LPS-induced in vitro model by simiR-128-3p group. ${ }^{\# \# ~}<0.01$ compared with LPS+Negative group. GAPDH=glyceraldehyde-3-phosphate dehydrogenase; LPS=lipopolysaccharide

of CSF ${ }^{[19,21]}$. Our study showed that miR-128-3p is a target spot of IncRNA AF131217.1 on the inflammation in vitro model.

KLF4 has been reported to physically bind to the active subunit P65 of transcription factor NF-KB in vascular endothelial cells to prevent the nuclear translocation of P65, thereby exerting its antiinflammatory role by inhibiting the transcriptional activation on downstream inflammatory factors of P65 ${ }^{[22]}$. In endothelial cells and immune cells, P300 can bind with the active subunit P65 of NF-KB to induce acetylation of $\mathrm{P} 65$, thereby promoting the transcriptional activation on downstream inflammatory factors by $\mathrm{P} 65^{[23]}$. Overexpressed KLF4 can competitively bind to P300, inhibiting the binding of P300 with P65 to attenuate its transcriptional activity, subsequently exerting an anti-inflammatory effect ${ }^{[23]}$. As members of the KLF family, KLF2 and KLF15 can also competitively bind with $\mathrm{P} 300^{[24]}$. It has been reported that KLF4 can bind to the active subunit P65 of NF-KB in vascular endothelial cells, to promote the binding of P65 to its downstream inflammatory factor vascular cell adhesion molecule 1, thereby playing a pro-inflammatory role ${ }^{[25]}$. Meanwhile, we found that KLF4 is a target spot of miR-128-3p on the inflammation in vitro model.

\section{CONCLUSION}

In summary, IncRNA AF131217.1 expression in the CSF model was activated and promoted inflammation by the suppression of miR-128-3p via KLF4/RhoF/NF-kB signal pathway, and this may 

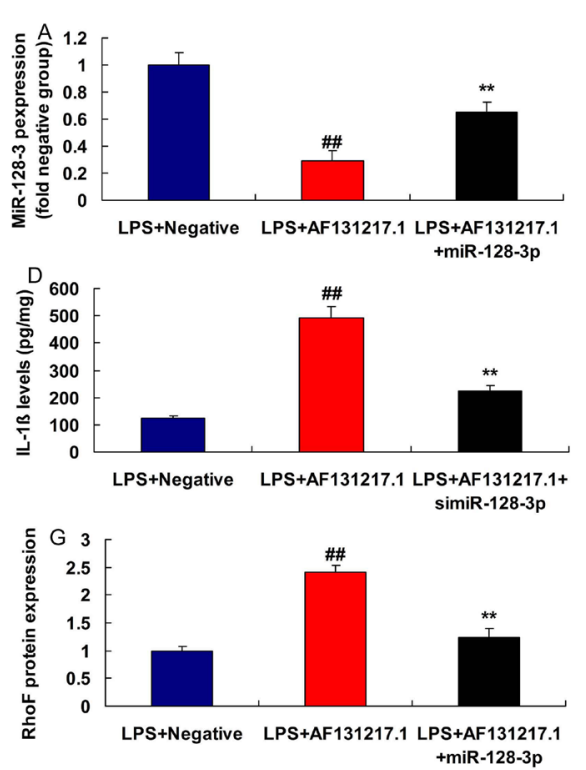
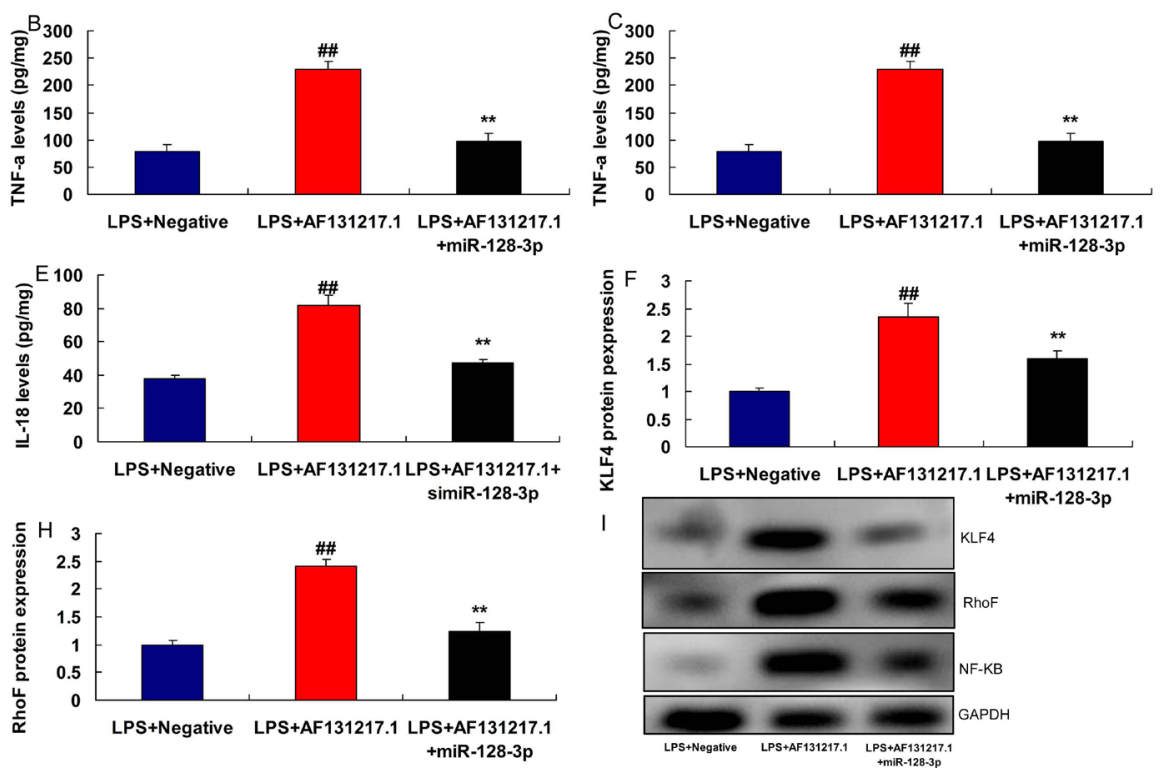

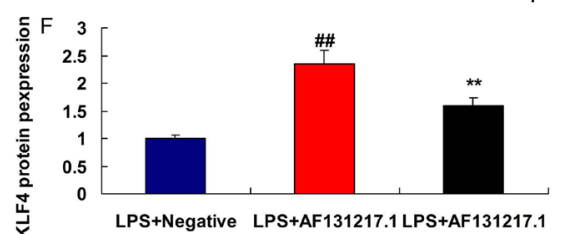

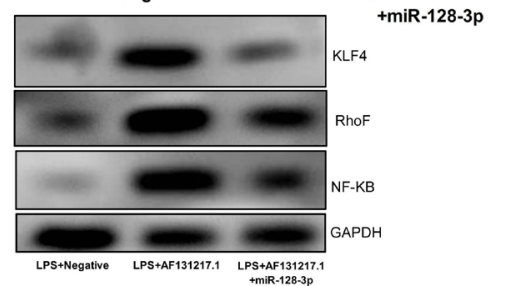

Fig. 7 - Long noncoding ribonucleic acid AF131217.1 regulated inflammation in the in vitro model via Kruppel-like factors (KLF) 4 by micro ribonucleic acid (miR)-128-3p. MiR-128-3p expression (A), tumor necrosis factor alpha (TNF-a), interleukin (IL)-6, IL-1B, IL-18, KLF4, RhoF, and nuclear factor kappa $B$ (NF-KB) protein expressions ( $B, C, D, E, F, G, H$, and I). LPS+Negative, LPS-induced in vitro model by negative mimics group; LPS+AF131217, LPS-induced in vitro model by AF131217.1 group; LPS+AF131217+miR-128-3p, LPS-induced in vitro model by AF131217.1 and miR-128-3p group. \#\# $P<0.01$ compared with $L P S+$ Negative group; ${ }^{*} P<0.01$ compared with $L P S+A F 131217.1$ group. GAPDH=glyceraldehyde3-phosphate dehydrogenase; $L P S=$ lipopolysaccharide

play an important role in the pathogenesis of CSF. An elevated plasma IncRNA AF131217.1 level may indicate the presence of CSF. Further studies are needed to establish clinical significance of increased plasma IncRNA AF131217.1 levels and to investigate the therapeutic efficacy of targeting miR-128-3p/KLF4/RhoF/ NF-KB signal pathway.

\section{Authors' roles \& responsibilities}

ZG Substantial contributions to the acquisition of data for the work; revising the work for important intellectual content; final approval of the version to be published

LZ Substantial contributions to the acquisition of data for the work; final approval of the version to be published

YY Substantial contributions to the acquisition of data for the work; final approval of the version to be published

XZ Substantial contributions to the analysis of data for the work; final approval of the version to be published

ZC Drafting the work and revising it; final approval of the version to be published

QW Revising the work; final approval of the version to be published

HJ Substantial contributions to the conception or design of the work; agreement to be accountable for all aspects of the work in ensuring that questions related to the integrity of any part of the work are appropriately investigated and resolved; final approval of the version to be published

\section{No financial support.}

No conflict of interest.

\section{REFERENCES}

1. Aydınlı B, Demir A, Özmen H, Vezir Ö, Ünal U, Özdemir M. Koroner Cerrahisinde Preoperatif HbA1c Değerleri Mortalite İcin Prediktor Olabilir mi? Turk J Anaesthesiol Reanim 2018; 46: 184-90. doi: 10.5152/ TJAR.2018.46667.

2. Almogati JG, Ahmed EO. Glycated Hemoglobin as a Predictor of the Length of Hospital Stay in Patients Following Coronary Bypass Graft Surgery in the Saudi Population. Braz J Cardiovasc Surg 2019; 34 (1): 28-32. doi: 10.21470/1678-9741-2018-0202.

3. Ouattara A, Lecomte P, Le Manach Y, Landi M, Jacqueminet S, Platonov I, et al. Poor intraoperative blood glucose control is associated with a worsened hospital outcome after cardiac surgery in diabetic patients. Anesthesiology 2005 October; 103: 687-94. doi: 10.1097/00000542-200510000-00006.

4. Lazar HL, Chipkin SR, Fitzgerald CA, Bao Y, Cabral H, Apstein CS. Tight glycemic control in diabetic coronary artery bypass graft patients improves perioperative outcomes and decreases recurrent ischemic events. Circulation 2004 March; 109:1497-502. doi: 10.1161 / 01.CIR.0000121747.71054.79

5. Peters AL, Davidson MB, Schriger DL, Hasselblad V. A clinical approach for the diagnosis of diabetes mellitus: an analysis using glycosylated hemoglobin levels. Meta-Research Group on the Diagnosis of Diabetes Using Glycated Hemoglobin Levels. JAMA 1996; 276: 1246-52. doi:10.1001/jama.1996.03540150048030. 
6. Tennyson C, Lee R, Attia R. Is there a role for HbA1c in predicting mortality and morbidity out comes after coronary artery bypass graft surgery? Interact Cardiovasc Thorac Surg 2013 December; 17(6): 1000-1008. doi: 10.1093/icvts/ivt351.

7. Faritous Z, Ardeshiri M, Yazdanian F, Jalali A, Totonchi Z, Azarfarin R. Hyperglycemia or high hemoglobin A1C: Which one is more associated with morbidity and mortality after coronary artery bypass graft surgery? Ann Thorac Cardiovasc Surg 2014; 20:223-8. doi: 10.5761/atcs.oa.13.02282.

8. Li Z, Amsterdam EA, Young JN, et al. Contemporary outcomes of coronary artery bypass grafting among patients with insulin-treated and non-insulin-treated diabetes. Ann Thorac Surg 2015 Dec;100(6):2262-9. doi: 10.1016/j.athoracsur.2015.06.028.

9. Najafi M, Goodarzynejad H. Determinants of length of stay in surgical ward after coronary bypass surgery: glycosylated hemoglobin as a predictor in all patients, diabetic or non-diabetic. JTehran Heart Cent. 2012 November;7(4):170-6. https://jthc.tums.ac.ir/index.php/jthc/ article/view/268

10. Medhi M, Marshall MC Jr, Burke HB, Hasan R, Nayak D, Reed G, et al. HbA1C predicts length of stay in patients admitted for coronary artery bypass surgery. HeartDis. Mar-Apr 2001;3(2):77-9. doi: 10.1097/00132580200103000-00003.

11. Khan MR, Khan H, Wahab A, Chaudharya S, Munirb A, et al. Effect of glycemic control on mortality and infections in patients undergoing coronary artery bypass grafting: a Genesee County experience. Journal of community hospital internal medicine perspectives 2019, vol. 9, no. 2, 74-79. doi: 10.1080/20009666.2019.1581044.

12. Okusa MD, Davenport A. Reading between the guidelines: the KDIGO practice guideline on acute kidney injury in the individual patient. Kidney Int. 2014;85(1):39-48. doi: 10.1038/ki.2013.378.

13. Latham R, Lancaster AD, Covington JF, Pirolo JS, Thomas CS. The association of diabetes and glucose control with surgical site infections among cardiothoracic surgery patients. Infect Control Hosp Epidemiol 2001; 22: 607-12. doi: 10.1086/501830.

14. Furnary AP, Gao G, Grunkemeier GL, et al. Continuous insulin infusion reduces mortality in patients with diabetes undergoing coronary artery bypass grafting. J Thorac Cardiovasc Surg. 2003; 125: 1007-1021. doi: 10.1067/mtc.2003.181.

15. Knapik P, Ciesla D, Filipiak K, Knapik M, Zembala M. Prevalence and clinical significance of elevated preoperative glycosylated hemoglobin in diabetic patients scheduled for coronary artery surgery. Eur J Cardiothorac Surg. 2011; 39(4): 484-9. doi: 10.1016/ j.ejcts.2010.07.037.
16. Matsuura K, Imamaki M, Ishida A, Shimura H, Niitsuma Y, Miyazaki M. Off-pump coronary artery bypass grafting for poorly controlled diabetic patients. Ann Thorac Cardiovasc Surg. 2009; 15(1): 18-22. http://www. atcs.jp/pdf/2009_15_1/18.pdf.

17. Kuhl J, Sartipy U, Eliasson B, Nyström T, Holzmann MJ. Relationship between preoperative hemoglobin A1c levels and long-term mortality after coronary artery bypass grafting in patients with type 2 diabetes mellitus. Int J Cardiol 2016; 202: 291-6. doi: 10.1016/j.jicard.2015.09.008.

18. Halkos ME, Puskas JD, Lattouf OM, Kilgo P, Kerendi F,Song HK, et al. Elevated preoperative hemoglobin A1c levelis predictive of adverse events after coronary artery bypass surgery. J Thorac Cardiovasc Surg September 2008; 136: 631-40. doi: 10.1016/j.jtcvs.2008.02.091.

19. Cohen O, Dankner R, Chetrit A, Luxenburg O, Langenauer C, Shinfeld A, et al. Multidisciplinary intervention for control of diabetes in patients undergoing coronary artery bypass graft(CABG). Cardiovasc Surg 2003; 11: 195-200. doi: 10.1016/s0967-2109(03)00019-x.

20. Beattie WS, Wijeysundera DN. Perioperative cardiac biomarkers: the utility and timing. Curr Opin Crit Care 2013; 19: 334-41. doi: 10.1097/ mcc.0b013e3283632f07.

21. Akram R. Allama, Magdy A. Sorourb, Mohamed M. Aghaa. Renal dysfunction after coronary artery bypass surgery. Research and Opinion in Anesthesia\&Intensive Care 2018, 5:103-109. doi: 10.4103/roaic. roaic_46_17.

22. Regner KR, Connolly HM, Schaff HV, Albright RC. Acute renal failure after cardiac surgery for carcinoid heart disease: incidence, risk factors, and prognosis. Am J KidneyDis 2005; 45: 826-832. doi: 10.1053/j. ajkd.2005.02.009.

23. Sevük U, Bilgiç A, Yaylak B, Ay N, Baysal E, Altındağ R, Alp V, Beyazıt Ü, Akkaya S, ErkulA. Relationship Between Elevated HbA1c and Deep Sternal Wound Infection in Patients Undergoing Cardiac Surgery. Koşuyolu Heart Journal 2016;19(1):7-11. doi: 10.5578/khj.10344.

24. Finger B, Brase J, He J, Gibson WJ, Wirtz K, Flynn BC. Elevated hemoglobin $\mathrm{A} 1 \mathrm{C}$ is associated with lower socioeconomic position and increased postoperative infections and longer hospital stay after cardiac surgical procedures. Ann Thorac Surg. 2017; 103(1): 145-51. doi:10.1016/j. athoracsur.2016.05.092.

25. Hadjinikolaou L, Klimatsidas M, Maria lacona G, Spyt T, Samani NJ. Short and medium term survival following coronary artery bypass surgery in British Indo-Asian and White Caucasian individuals: impact of diabetes mellitus. Interact Cardiovasc Thorac Surg 2010; 10: 389-93. doi: 10.1510/icvts.2009.210567.

533 